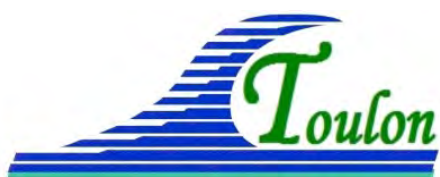

XIV èmes Journées Nationales Génie Côtier - Génie Civil

Toulon, 29 juin au $1^{\text {er }}$ juillet 2016

DOI:10.5150/jngcgc.2016.033 @ Editions Paralia CFL

disponible en ligne - $h$ ttp://www.paralia.fr - available online

\title{
Evaluation des stocks sédimentaires littoraux : prospection par sismique réflexion haute-résolution sur le littoral varois
}

\author{
Alexis STEPANIAN ${ }^{1}$, Isabelle THINON ${ }^{2}$
}

1. BRGM, Direction Régionale Provence Alpes Côte d’Azur, BP 168, 13276 Cedex 09, Marseille, France. a.stepanian@brgm.fr

2. BRGM, Direction des Géoressources, Unité Géologie des Bassins Sédimentaires, 3 Avenue Claude Guillemin, 45100 Orléans, France.

i.thinon@brgm.fr

\section{Résumé :}

Le Syndicat Mixte du SCoT Provence-Méditerranée et le BRGM ont établi un partenariat pour disposer d'une connaissance actualisée des risques littoraux à travers un projet de caractérisation des aléas érosion côtière et submersion marine sur les communes varoises entre Saint-Cyr-sur-Mer et Le Lavandou. L'évaluation quantitative des volumes sédimentaires sur le proche avant-côte est une donnée importante pour caractériser le potentiel de résilience des plages face aux phénomènes érosifs à différents pas de temps et pour apporter des solutions de gestion. Dans l'objectif d'obtenir une estimation des stocks sédimentaires sur 15 secteurs le long du littoral varois, une campagne de prospection sismique très-haute-résolution, nommée VARSISCOT, s'est déroulée en octobre 2014. L’évaluation des épaisseurs de sédiments met en évidence une grande variabilité avec des valeurs maximales de près de $8 \mathrm{~m}$. Même si l’âge des dépôts reste indéterminé, on observe une certaine cohérence entre les zones amaigries/riches et le sens prédominant de la dérive littorale actuelle. Les données originales recueillies permettent de satisfaire les objectifs de production d'un état initial de la connaissance des épaisseurs sédimentaires sur les avant-plages et d'estimation du potentiel de résilience naturelle des plages face à une érosion chronique. Les exemples pris de part et d'autre de la presqu'île de Giens éclairent également d'une manière inédite l'architecture sous-marine des prismes sédimentaires côtiers d'un secteur du littoral à haute valeur touristique et patrimoniale.

Mots-clés : Prisme littoral, Géophysique marine, Sismique réflexion, Géologie quaternaire.

\section{Introduction}

Dans le cadre de l'élaboration du volet littoral et maritime du SCoT Provence Méditerranée (SCoT PM, figure 1), les communes littorales varoises entre Saint-Cyrsur-Mer et Le Lavandou ont décidé de mettre en œuvre une étude diagnostique des risques côtiers sur leur territoire. Le Syndicat Mixte du SCoT Provence-Méditerranée et 


\section{Thème 2 - Dynamique sédimentaire}

le BRGM ont établi un partenariat pour disposer d'une connaissance actualisée des risques littoraux à travers un projet de caractérisation des aléas érosion côtière et submersion marine sur l'ensemble des communes adhérentes. La question des volumes des stocks sédimentaires sableux littoraux est abordée à travers d'une part leur quantification et d'autre part leur utilisation potentielle en termes de technique de gestion de l'érosion et du recul du trait de côte associé. Le projet vise à l'acquisition de profils de sismique très-haute-réflexion (THR) dans les très petits fonds entre le trait de côte et l'herbier de posidonies dont la profondeur et la proximité de la côte est très variable sur le périmètre de l'étude. De telles données permettent d'imager le sous-sol et ainsi d'accéder à une estimation des épaisseurs sédimentaires sur les avant-plages. Cet article présente les résultats de l'interprétation des données sismiques THR VARSISCOT, acquises en octobre 2014. Cette connaissance, inédite au niveau des avants-plages du littoral des communes concernées, est ici illustrée à travers des exemples pris le long du double tombolo de Giens qui constitue un trait morphologique majeur du littoral varois.

\section{Contexte sédimentaire du plateau continental}

Le plateau continental varois est relativement étroit à l'exception de la rade d'Hyères (figure 1). Une rupture de pente entre -90 et $-100 \mathrm{~m}$ marque le rivage tel qu'il était lors du dernier maximum glaciaire, il y a environ 18000 ans BP. En période glaciaire, le plateau continental, alors exondé, était parcouru par les fleuves et les rivières qui y ont déposé les produits de l'érosion des massifs rocheux continentaux. Lors de la remontée du niveau marin au cours de l'Holocène, la mer a remobilisé les dépôts, en particulier les sables, du plateau continental vers le littoral, l'alimentation par les fleuves se poursuivant à un rythme différent, en volume et en nature, en fonction des variations climatiques. La nature et la répartition des sédiments sur le plateau résultent ainsi d'une histoire géologique ancienne. Cependant, elle reste également influencée par les agents de forçages actuels, houle et courants, en particulier dans la zone littorale. Les données existantes sur les épaisseurs sédimentaires du plateau continental sont issues des investigations menées dans les années 1970 par le CNEXO pour la recherche de granulats marins qui ont montré que les gisements potentiels (Bandol, Rade d'Hyères, Bormes) étaient rares, et soumis à des contraintes environnementales (BLANC et al., 1972 ; LEENHARD, 1972). Les sédiments y sont majoritairement sableux, avec une composante plus ou moins forte de matériaux fins, et leur exploitation était fortement déconseillée en raison des impacts probables sur l'état écologique de l'herbier de posidonies et par voie de conséquences, à terme sur la stabilité du trait de côte (BLANC, 1975 ; SOGREAH, 1984). 


\section{XIV ${ }^{\text {èmes }}$ Journées Nationales Génie Côtier - Génie Civil \\ Toulon, 29 juin au $1^{\text {er }}$ juillet 2016}

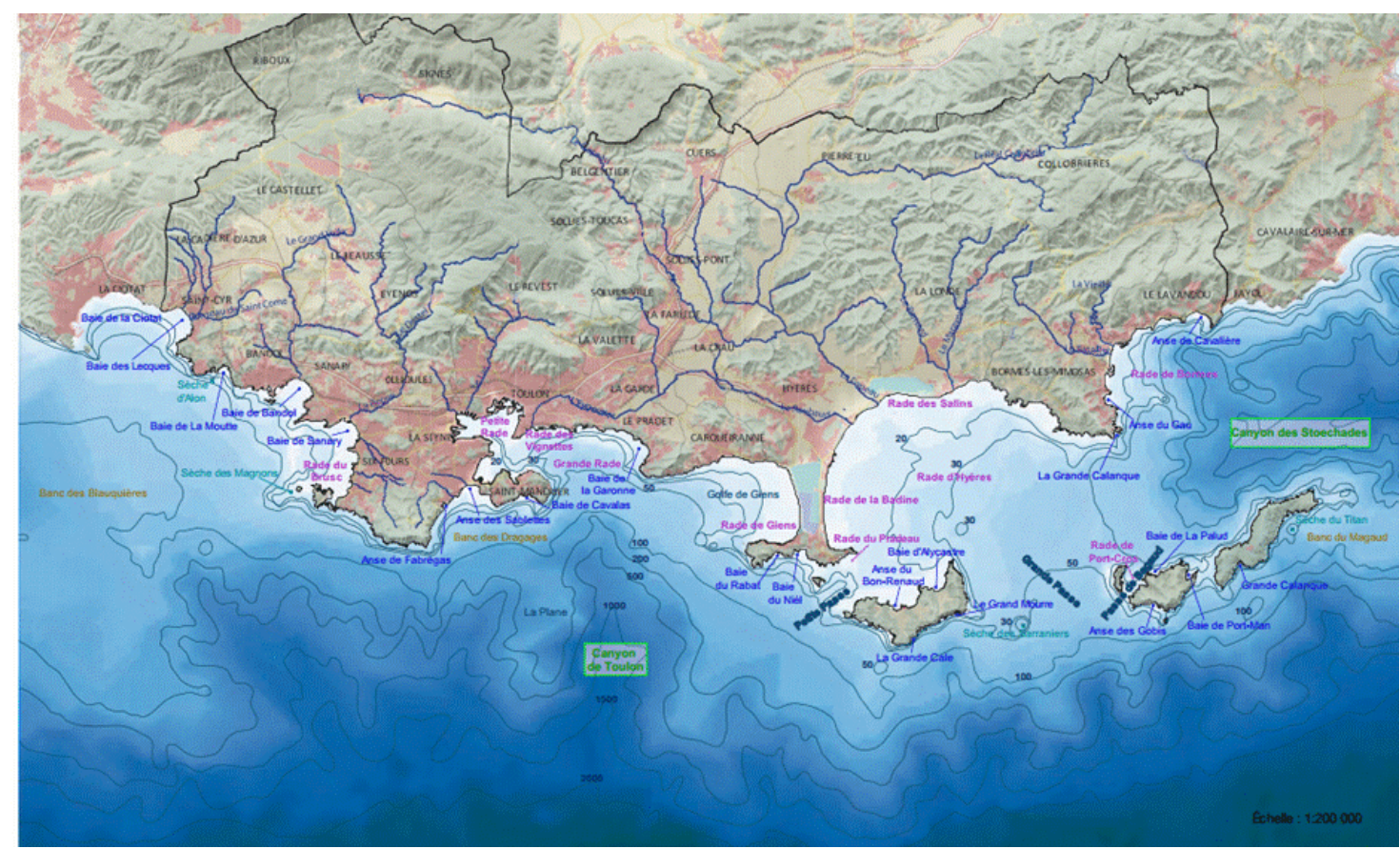

Figure 1. Présentation générale du littoral du SCoT Provence-Méditerranée (Source : Agence d'Urbanisme de l'Aire Toulonnaise).

Dans les petits-fonds, les données sont inexistantes, et seules certaines plages de poches de Porquerolles ont déjà fait l'objet de prospection géophysique (BRUNEL, 2010) : le prisme littoral y est peu épais, et sa répartition est contrainte par la géométrie du substratum rocheux sous-jacent. C'est de plus une masse sableuse relique plus du tout alimentée par des cours d'eau côtiers.

\section{Données et méthodologies}

Quinze zones cibles pour une prospection géophysique ont été identifiées d'après un inventaire des stocks sédimentaires présents dans la zone côtière, effectué sur la base des données existantes (STEPANIAN, 2014). La mission d'acquisition de sismique réflexion THR VARSISCOT s'est déroulée entre le 24 et le 30 octobre 2014 entre Le Lavandou, à l'Est, et le Cap Sicié à l'Ouest (figure 2). Sur chacune des zones cibles, un maillage de profils orthogonaux au trait de côte a été réalisé avec un espacement interprofils de 50 à $200 \mathrm{~m}$ en fonction de la configuration locale du littoral et de l'extension de la zone d'exploration. Environ $130 \mathrm{~km}$ de profils sismiques THR ont été acquis à l'aide d'un boomer de type IKB Seistec (BALTZER et al., 2006 ; 2008 \& 2014) à vitesse de 2 à 3 noeuds. Le dispositif se présente sous forme d'un catamaran sur lequel sont fixés un émetteur et un récepteur à géométrie fixe (figure 3). 


\section{Thème 2 - Dynamique sédimentaire}

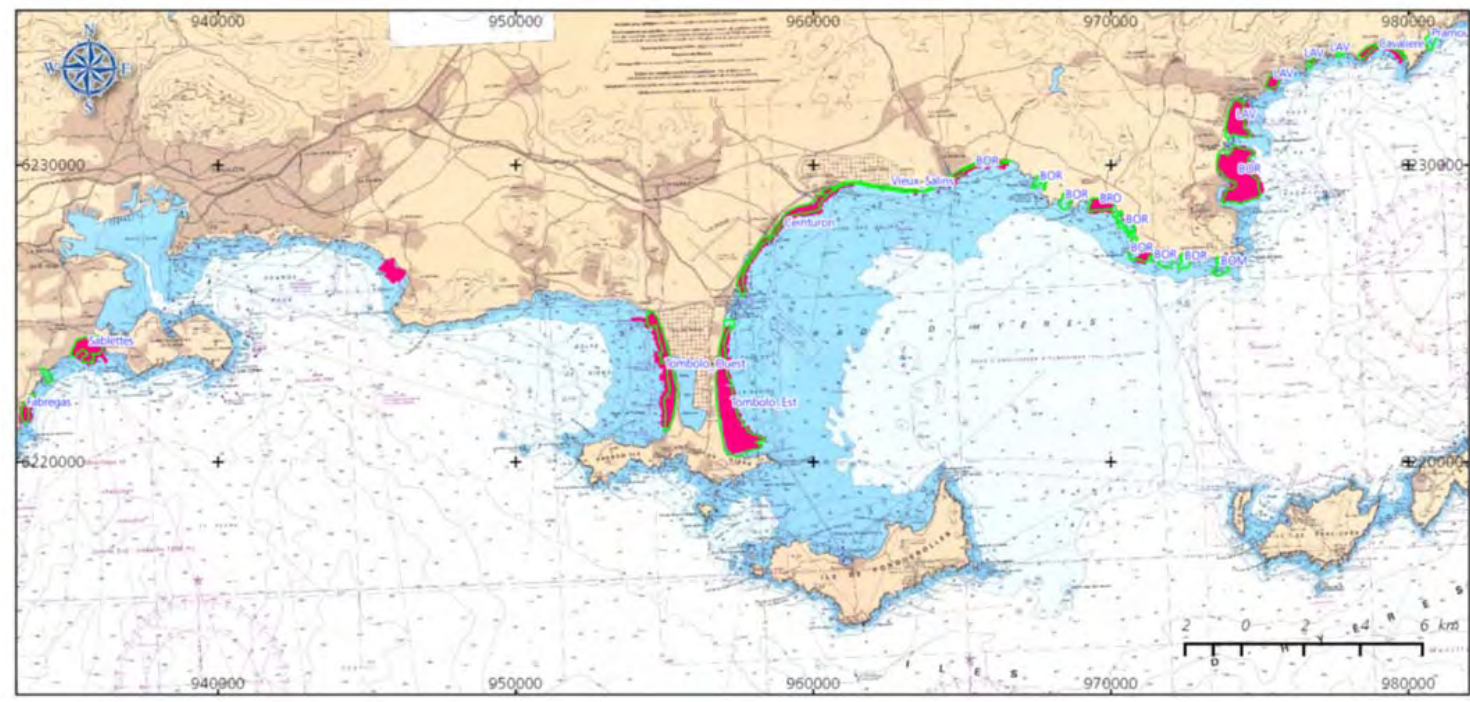

Figure 2. Plan de positionnement de l'acquisition géophysique et zone pressentie (fond : carte marine - (C) SHOM).

Le Boomer est adapté aux très faibles profondeurs d'eau et aux zones de petites dimensions (baies, zones portuaires, ...). Il permet une prospection à très haute résolution verticale (décimétrique) et latérale (métrique). La longueur d’imagerie est limitée par la présence du multiple primaire (echo), qui est la répétition du signal au temps double et masque ainsi l'image sismique sous-jacente. Elle est aussi fonction de la lithologie rencontrée. La pénétration acoustique est supérieure à $20 \mathrm{~m}$ dans les couches vaseuses et sableuses. Le sous-sol est aussi imagé sous les zones d'herbiers, à condition que la matte soit peu importante. Les conditions de navigation et d'acquisition ont été relativement satisfaisantes sur l'ensemble de la campagne. Il est à noter cependant la présence d'une houle de hauteur d'environ $0,5 \mathrm{~m}$ pour des périodes de vagues courtes (3-5 s).

Le traitement sismique sous Seismic Unix ${ }^{\circledR}$ (COHEN \& STOCKWELLL, 2004) des données sismiques VARSISCOT a consisté principalement à corriger la géométrie du système d'acquisition et les effets de la houle (MARY, 2004), à appliquer des filtres passband afin d'améliorer le rapport signal sur bruit. Afin de faciliter l'interprétation sismique numérique sous le logiciel GEOGRAPHIX ${ }^{\circledR}$, les profils sismiques ont été dilatés (temps multiplié par 10). L'axe vertical des profils VARSISCOT est en $10^{-3}$ seconde temps double. L'axe horizontal est en tir (la distance entre deux tirs est de 1 et $2 \mathrm{~m}$ ). Les profondeurs et épaisseurs en temps dérivées des interprétations sismiques ont été transformées en profondeur en prenant une vitesse moyenne de propagation de $1700 \mathrm{~m} / \mathrm{s}$. L’incertitude totale portant sur les épaisseurs est estimée de l'ordre de 40 à $50 \mathrm{~cm}$. 


\section{XIV èmes Journées Nationales Génie Côtier - Génie Civil \\ Toulon, 29 juin au $1^{\text {er }}$ juillet 2016}
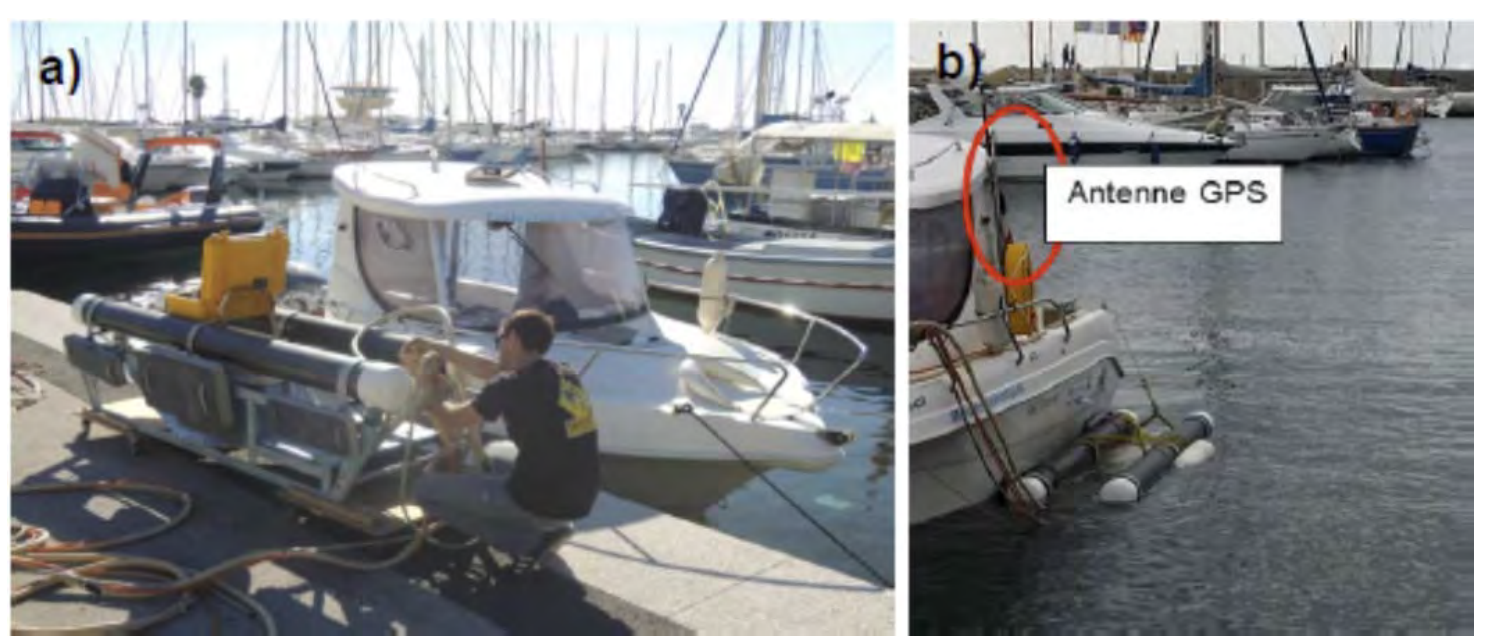

Figure 3. Dispositif Seistec a) à quai et b) à couple du support de navigation en position d'acquisition. L'antenne GPS assure le positionnement en x,y de la donnée sismique.

\section{Résultats}

Les épaisseurs mesurées sont très variables en fonction des sites. Sur l'ensemble des sites investigués, elles varient entre $40 \mathrm{~cm}$ et 8-9 $\mathrm{m}$ au maximum.

Les résultats sont illustrés ici sur deux secteurs de part et d'autre du tombolo de Giens. Les cartes des isopaques pour les façades sont reportées sur la figure 4. Les masses sédimentaires le long du tombolo Ouest sont plus faibles en volume et en surface par rapport aux fortes épaisseurs évaluées sur le tombolo Est.

Le long du tombolo Ouest, la répartition des masses sédimentaires est assez variable et traduit probablement la complexité des transits sédimentaires et hydrodynamiques de cette portion du littoral décrits par BLANC (1975) et COURTAUD (2000) avec (i) des transferts sableux bidirectionnels Nord-Sud et Sud-Nord dans l'ensemble de la cellule sédimentaire, et (ii) des courants d'arrachements, dirigés vers le large qui peuvent mettre à l'affleurement des dépôts indurés anciens et inciser l'herbier de posidonies. L'extrait de profil TW270 montre l'amincissement du prisme sédimentaire depuis la côte vers le large. La zone acoustique sourde au droit de l'herbier masque la prolongation du substratum rocheux sous celui-ci. On distingue également une unité supérieure bien litée, probablement de sables fins qui reposent probablement sur des sédiments plus fins mais plus indurés (vases et/ou tourbes). Ces dépôts fins correspondent très probablement à l'ancienne extension latérale de la lagune littorale, convertie en zones de production saline au cours du XIX ${ }^{\mathrm{eme}}$ siècle. 


\section{Thème 2 - Dynamique sédimentaire}

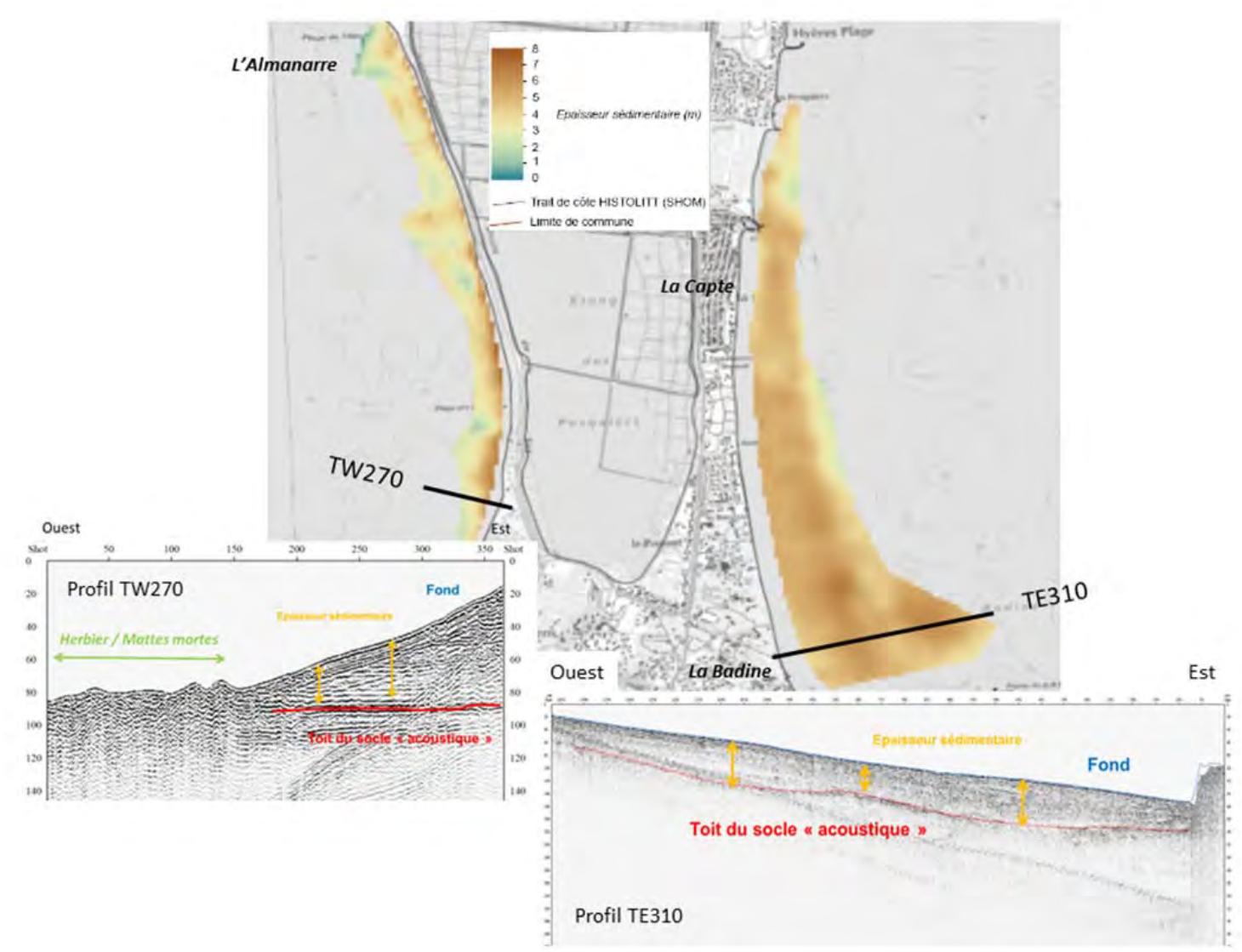

Figure 4. Carte des isopaques de sédiments le long du double tombolo, et exemple de profils sismiques sur le tombolo Ouest et Est.

Sur le tombolo Est, l'accumulation sédimentaire au Sud et la décroissance progressive de son épaisseur vers le Nord et le port d'Hyères traduisent la dérive littorale Nord- Sud prévalant sur cette portion. Le déficit sédimentaire au Nord traduit également clairement le rôle de blocage des transits sédimentaires que jouent les jetées du Port d'Hyères. Le transfert résiduel vers le Sud contribue à l'appauvrissement progressif du budget sédimentaire des plages situées directement en aval-dérive au Sud du Port (Anse de la Potinière, Bona, etc.), au niveau du trait de côte, mais également au niveau du prisme littoral dans les petits-fonds. A l'inverse, on ne constate pas de diminution significative de l'épaisseur des stocks sableux dans les petits-fonds au droit de la plage de la Capte, qui est pourtant en érosion chronique. Il apparait ici des relations complexes entre la plage et le prisme sédimentaire qui nécessitent des investigations locales sur les transits sableux. Enfin, la disposition des masses sédimentaires dans la zone sous-marine de part et d'autre du double tombolo laisse supposer que la morphologie actuelle de celui-ci n’est pas en équilibre avec les conditions hydrosédimentaires qui distribuent les volumes sableux. Ainsi, on peut supposer qu'en l'absence d'aménagement du double 


\section{XIV èmes Journées Nationales Génie Côtier - Génie Civil \\ Toulon, 29 juin au $1^{\text {er }}$ juillet 2016}

tombolo (salines, étangs, routes, etc.), qui contraignent artificiellement sa géométrie, l'orientation du double tombolo, aujourd'hui Nord-Sud, serait probablement différente avec une orientation Est plus marquée.

\section{Conclusions}

La campagne sismique THR VARSISCOT s'est déroulée en octobre 2014 sur une série de zones cibles définies au préalable sur des critères bibliographiques et d'intérêts prioritaires en termes d'usages potentiels de la ressource sédimentaire. Les objectifs principaux de cette évaluation étaient de produire un état initial de la connaissance des épaisseurs sédimentaires sur les avant-plages, et d'avoir une estimation du potentiel de résilience naturelle des plages face à une érosion chronique. L'évaluation des volumes et des épaisseurs sédimentaires sur les 15 sites prospectés met en évidence une grande variabilité du disponible sédimentaire avec des épaisseurs variant de $40 \mathrm{~cm}$ à près de $8 \mathrm{~m}$. L’âge des dépôts reste inconnu en l'absence de données de carottages, mais une cohérence est observée entre les zones amaigries/riches et le sens prédominant de la dérive littorale actuelle. Cela suggère des conditions de dépôts à long-terme comparables à la situation actuelle. La rade d'Hyères et le double tombolo pris en exemple dans cet article se distingue de ce modèle dans la mesure où la distribution des zones en accrétion et érosion est principalement conditionnée par les impacts sur le transit littoral des ouvrages transversaux (jetées des ports, épis, digue), qui ont été implantés principalement depuis les années 70. La présence d'un stock sableux sur l'avant-côte est indispensable à la résilience d'une plage aux phénomènes érosifs ponctuels lors de tempêtes marines, pour favoriser une reconstruction naturelle du profil de plage pendant la période post-tempête, et diminuer ainsi la vulnérabilité du site à l'érosion et au recul du trait de côte (ex : anse de la Badine, tombolo Est). A l'inverse, des sites, à recul modéré du trait de côte, présentent un stock très limité sur l'avant-côte, et donc une plus grande vulnérabilité (ex : tombolo Ouest).

Enfin, les données sismiques THR acquises durant la campagne VARSISCOT constituent une source d'information inédite sur la structure géologique locale du prisme sédimentaire côtier, dont l'exploitation est susceptible d'alimenter les réflexions lors de futurs projets d'aménagement du littoral.

\section{Remerciements}

Ce travail a été cofinancé par le BRGM et le Syndicat Mixte du SCoT ProvenceMéditerranée, avec le soutien de l'Europe (FEDER), du Conseil Régional ProvenceAlpes-Côte d'Azur et du Conseil Départemental du Var. Il a bénéficié de l'aide technique de l'Agence d'Urbanisme de l'Aire Toulonnaise AU[dat]. Les auteurs remercient Caroline Labaune et les membres de la société CREOCEAN, et de l'EID Méditerranée pour la mise en œuvre du matériel de prospection géophysique. 


\section{Références bibliographiques}

BALTZER A., STEPANIAN A., BOUTIN R. (2006). Apport de la sismique très haute résolution en domaine portuaire : identification des structures géologiques et tectoniques sous-marines. Journées Nationales Génie Côtier - Génie Civil, Brest, pp 511-517. http://dx.doi.org/10.5150/ingcgc.2006.049-B

BALTZER A., BOUTIN R., STEPANIAN A. (2008). Projets portuaires : apport de la géophysique marine très haute résolution. Génie Côtier - Génie Civil, SophiaAntipolis, pp 677-686. http://dx.doi.org/10.5150/jngcgc.2008.064-B

BALTZER A., MAANAN M., ROLLO N., DURAND A (2014). Approche géophysique de la couverture sédimentaire au large des Pays de Monts. Journées Nationales Génie Côtier - Génie Civil, Dunkerque, pp 539-546. http://dx.doi.org/10.5150/ingcgc.2014.059

BLANC J.J. (1975). Recherches de sédimentologie appliquée au littoral rocheux de la Provence. Aménagement et protection. CNEXO, 164 p.

BLANC J.J., PICARD F., FRANZA M., JEUDY DE GRISSAC A. (1972). Recherches sur les gites sous-marins de sables et de graviers sur le précontinent de la Provence occidentale. Rapport CNEXO, 72/475, $42 \mathrm{p}$.

BRUNEL C. (2010). Evolution séculaire de l'avant-côte de la Méditerranée française. Thèse de Doctorat, Université Aix-Marseille I, 470 p.

COHEN J.K., STOCKWELL JR. J.W., (2004). CWP/SU: Seismic Unix Release No. 38: a free package for seismic research and processing. Center for Wave Phenomena, Colorado School of Mines.

COURTAUD J. (2000). Dynamiques géomorphologiques et risques littoraux. Cas du tombolo de Giens. Thèse Université Aix Marseille 1, 265 p.

LEENHARD O. (1972). Sables et graviers entre l'embouchure du Rhône à la frontière italienne. Musée Océanographique de Monaco, Contrat CNEXO, 72/471, 6 p.+ cartes.

MARY J.F. (2004). Traitement du signal en sismique réflexion très haute résolution. Rapport de stage Licence IUP2 Centre Littoral de Géophysique, Université de La Rochelle.

SOGREAH (1984). Catalogue sédimentologique des côtes françaises. Côtes de la Méditerranée. Collection de la Direction des Etudes et Recherches d’EDF, Ed. Eyrolles, $288 \mathrm{p}$.

STEPANIAN A. (2014). Evaluation des Risques Naturels Littoraux - SCoT ProvenceMéditerranée - Evaluation des stocks sédimentaires littoraux, diagnostic préliminaire. Rapport final. BRGM/RP-63951-FR, 81 p., 29 ill., 4 ann., 1 CD. 